\title{
Settling slurry flow near deposition velocity in inclined pipe of negative slope
}

\author{
Mikoláš Kesely ${ }^{1 *}$, Václav Matoušek ${ }^{1}$, Pavel Vlasák ${ }^{1}$ \\ ${ }^{1}$ Institute of Hydrodynamics of Czech Academy of Sciences, Pod Patankou 30/5, 16612 Prague 6, Czech Republic
}

\begin{abstract}
Pipe flow of sand-water slurry (settling slurry) is sensitive to pipe inclination. The effect of the angle to which the partially stratified flow is inclined from the horizontal has been subject to investigation in numerous studies. However, almost all of them focus on ascending flows, i.e. flows inclined to positive angles of inclination. It is well known that settling slurry flows inclined to negative slopes (descending flows) differ from those inclined to positive slopes, particularly at velocities near the deposition limit. The deposition limit velocity is the flow velocity at which stationary deposit starts to be formed at the bottom of the pipe. We investigate the effect of the negative slope on pipe flow near deposition limit velocity in the broad range of inclination angles. Besides the deposition limit, we focus on the distribution of solids across the pipe cross section. We combine experimental approach with mathematical modelling. Our new experiments with medium-to-coarse sand (mass-medium grain size $0.87 \mathrm{~mm}$ ) in a $100-\mathrm{mm}$ pipe inclined from 0 to -45 degree provide suitable data for a validation of predictions of our layered model for partially stratified flows in inclined pipes.
\end{abstract}

\section{Introduction}

Pipeline systems transporting settling slurries are composed of horizontal sections with vertical raisers and depending on operational conditions, inclined sections of various lengths over broad range of angles. When such pipeline system is evaluated, effect of inclined sections on design parameters should be taken into account. A broad range of pipe inclinations produce slurry flows of different behaviour and properties.

Our research showed [1] that the distribution of solids across the pipe cross section is sensitive to the angle of inclination, which has an effect on both the pressure drop and deposition limit velocity. Despite its practical importance, the effect of pipe inclination on these parameters has received little attention in research. Experimental results are extremely scarce in the literature, almost non-existent in the case of negative slopes.

This paper is focused on the settling slurry flow and the effect of negative slopes on its partially stratified structure. The effect of inclination is investigated by both mathematical and physical modelling and the aim is to verify our layered model by comparing its results with suitable experimental data.

\section{Predictive modelling approach}

To predict the behaviour of partially stratified settling slurry flow in inclined flows, we use our new layered model [2]. The model consists of two layers (the lower contact bed layer formed by bed of particles and the upper layer) with the interfacial sub-layer called a shear layer linking the two layers together. For the lower layer it is assumed, that the particles are in constant mutual contact with uniform distribution of grains across the bed. Depending on the hydraulic conditions of the flow, the model calculates, whether the lower layer slides over the pipe wall, or forms a stationary bed. The layered model can be therefore used to calculate the value of deposition limit velocity $V_{D}$ for inclined flows. For the negative slopes, the calculation is limited by the particle sliding friction angle, as the model equations do not converge to any solution when the sliding bed velocity is higher than the velocity of flow above the bed. Hence, it is not applicable for steep flows, where the sliding of the bed is dominated by gravitational forces. The interfacial shear layer contains colliding grains in sporadic contact. The distribution of colliding grains is considered to be linear across the shear layer.

The model inputs are pipe parameters (the internal diameter, the wall roughness and the angle of inclination of the pipe), properties of liquid and solids (liquid and solids density, particle size), the model constants $\left(\mu_{s}=\right.$ coefficient of sliding friction, $\tan \varphi=$ the coefficient of internal friction of colliding grains in the shear layer, $K=$ the ratio of local horizontal and vertical components of solids stress in the bed layer), the cross-section averaged velocity of flow, $V_{m}$, the solids concentration in the contact layer, $C_{2}$, and the other of the two average concentrations (either the spatial concentration $C_{v i}$ or the delivered concentration $C_{v d}$ ). For these inputs, the model calculates the solids distribution (the thickness of both, the lower contact bed layer and the shear layer), pressure

* Corresponding author: kesely@ih.cas.cz 
drop, sliding bed velocity and the other of the two overall concentrations $\left(C_{v i}\right.$ or $\left.C_{v d}\right)$

\section{Experimental modelling of sand slurry flow}

In order to evaluate our predictive modelling approach, we carried out experimental measurements with fraction of medium to coarse sand in $100 \mathrm{~mm}$ pipe loop at Institute of Hydrodynamics of the Czech Academy of Sciences in summer of 2017 and 2018. The experimental set-up, methods and results are described in [3, 4] The pipe loop contains a tilting section, which can be inclined to an arbitrary slope between horizontal and vertical. The tests were carried out in various slopes from 0 to -45 degrees. Manometric pressure differences (DPT) were measured by differential pressure transmitters, in several measuring sections along the loop together with average velocity of the flow sensed by a magnetic flow meter in a vertical pipe section of the loop. Tests runs at different pipe tilts included parallel measurements of concentration profiles across a pipe cross section. Delivered concentration of solids at different flow velocities was determined in the vertical sections using DPT measurements.

The tested fraction was a narrow-graded fraction of sand (code SP090, $d_{50}=0.87 \mathrm{~mm}, \rho_{s}=2620 \mathrm{~kg} / \mathrm{m}^{3}$ ). Two constant values of solids volumetric concentrations were used. One produced maximum value of $C_{v d}$ of 0.26 and the other maximum value of 0.37 . Experimental data with medium to coarse sand as SP090 are insufficiently represented in the literature database.

\subsection{Experimental identification of deposition limit velocity}

Deposition limit velocity was determined by visual observation using cameras, which were aimed at the bottom of the pipe. By definition, deposition limit velocity is the flow velocity at which stationary deposit starts to be formed at the bottom of the pipe. Unfortunately, experiments showed very unsteady flow of solids, when mean velocity of the flow approached region around the value of $V_{D}$. The observed $V_{m}$ range within which particles stopped sliding was rather broad. Just to get some sense of values, first particles interrupted sliding at $V_{m}$ about $2.0 \mathrm{~m} / \mathrm{s}$, while a steady state deposit developed below $0.7 \mathrm{~m} / \mathrm{s}$. At velocities between these limits, the bed interrupted sliding regularly for a period of time. The ratio between time periods, when the bed was stationary and when was sliding proved to be dependent on the value of $V_{m}$. With decreasing mean velocity of the flow, time periods of stationary bed grew longer. We call this behaviour a „caterpillar behaviour" of the sliding bed. The value of deposition limit velocity was assumed to a ratio of period roughly equal to $10 / 90$ (sliding bed/stationary bed).

\section{Discussion of results}

\subsection{Distribution of solids concentration}

In Figures 1 and 2, measured concentration profiles are plotted for the two different values of volumetric concentrations in pipeline and mean flow velocity roughly $1.5 \mathrm{~m} \cdot \mathrm{s}^{-1}$. Both figures show that the flow is stratified with a steep concentration gradient. Furthermore, two strong effects of pipe inclination on flow patterns can be identified.

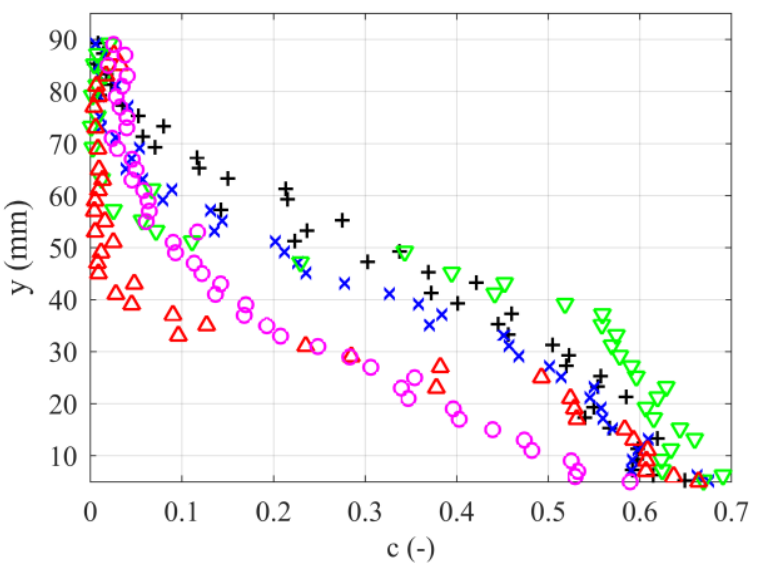

Fig. 1. Distribution of granular concentration at $V_{m} \sim 1.5 \mathrm{~m} \cdot \mathrm{s}^{-1}$ for flow of SP090 slurry $\left(C_{v d, \max }=0.26\right)$ at different pipe inclination (descending pipe). Legend: black $=0 \mathrm{deg}$, blue $=$ $-15 \mathrm{deg}$, green $=-25 \mathrm{deg}$, red $=-35 \mathrm{deg}$, magenta $=-45 \mathrm{deg}$.

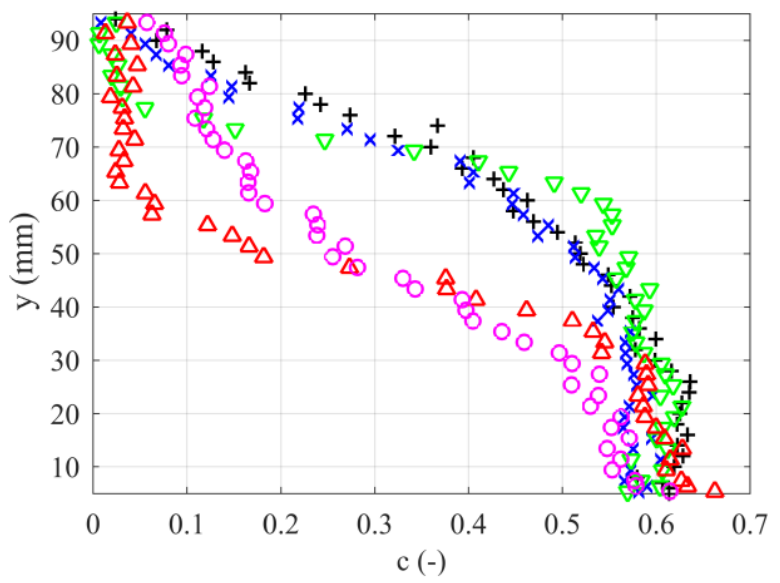

Fig. 2. Distribution of granular concentration at $V_{m} \sim 1.5 \mathrm{~m} . \mathrm{s}^{-1}$ for flow of SP090 slurry $\left(C_{v d, \max }=0.37\right)$ at different pipe inclination (descending pipe). Legend: black $=0 \mathrm{deg}$, blue $=$ $-15 \mathrm{deg}$, green $=-25 \mathrm{deg}$, red $=-35 \mathrm{deg}$, magenta $=-45 \mathrm{deg}$.

Firstly, the delivered concentration of particles gets diminished at higher angles of inclination although the flow velocity remains approximately the same. This is due to formation of the unsteady bed (caterpillar behaviour) in the ascending section of the pipe loop and this bed moves slower if the inclination angle increases. 
As a result of this, the spatial concentration increases in the ascending pipe while it decreases in the descending section of the loop. For instance, much thicker sliding bed and hence higher cross-section average spatial concentration is observed in the pipe inclined to -25 degree than in the same pipe to -35 or -45 degree (Figures 1 and 2).

Secondly, it is interesting to observe how the degree of stratification varies with the negative angle of pipe inclination. According to Figures 1 and 2, the flow is the most stratified at inclination angles -25 and -35 degree. At steep inclinations, the flow tends to be less stratified with almost non-existent contact layer as the sliding bed becomes much thinner and many particles is transported in flow above the bed. At mild inclinations, say up to -15 degree, the flow maintains the shear layer of the thickness similar to the one in the horizontal pipe. The reason why the thickness of the shear layer diminishes if the angle exceeds -15 and virtually disappears at angles of about -25 and -35 degree for our tested material is the faster sliding bed in more inclined pipe. As a result of this, the velocity difference between the flow above the bed and the sliding bed itself diminishes and so diminishes the shear stress at the top of the bed responsible for the development of the shear layer.

The discussed effects of pipe inclination on the distribution of solids concentration has many consequences for the flow behaviour. One of those is the effect on the establishment of stationary deposit at the deposition limit velocity in a pipe. We want to test abilities of our layered model to capture the observed variations in the concentration distribution with pipe inclination and to predict the deposition limit velocity for the observed flow conditions in a negatively sloped pipe.

\subsection{Flow near deposition limit velocity}

We compare our model predictions of the concentration distribution across a pipe cross section with experimental results for the SP090 slurry flow inclined to different negative slopes up to -45 degree. In the model, we use the measured $V_{m}$ and $C_{v d}$ as model inputs. The model constants are set to $K=0.50, \tan \varphi=0.75, \mu_{s}=0.49$, the bed concentration $C_{2}$ varies from 0.5 to 0.6 depending on flow conditions.

The concentration profiles were measured at flow velocities slightly above the deposition limit velocity and they serve here to evaluate the accuracy of layered model predictions for various pipe inclinations and average solids concentrations.

Figures 3 to 6 show concentration profiles in the SP090 slurry flow at certain flow velocity near deposition limit velocity for two different angles of inclination ( -25 and -15 degree) and slurry of inline solids concentration of about 0.26 (Figures 3 and 4 ) and a higher inline concentration of about 0.37 (Figures 5 and 6). In plots of Figures 3 to 6 , the measured profiles are compared with simplified profiles predicted by the layered model.

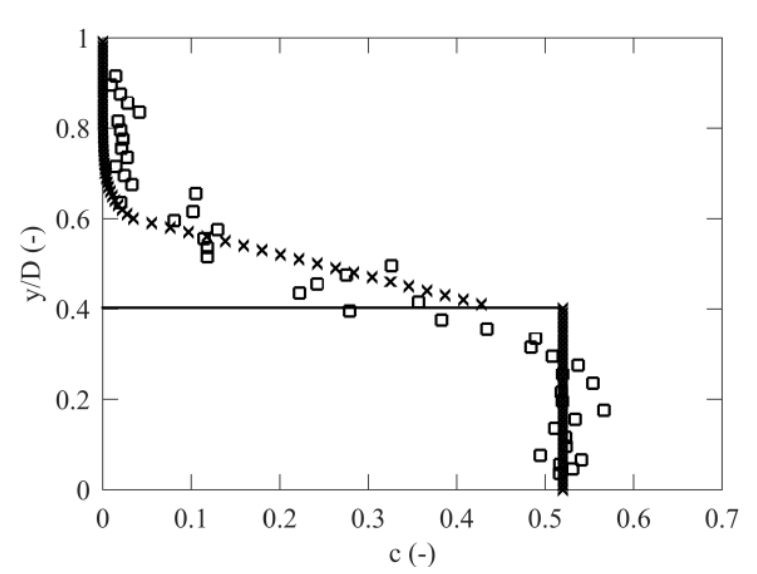

Fig. 3. Distribution of granular concentration at velocity slightly above deposition limit for flow of SP090 slurry $\left(C_{v d, \text { max }}=0.26\right)$ at pipe inclination of $-15 \mathrm{deg}, V_{m}=1.1 \mathrm{~m} . \mathrm{s}^{-1}$, $C_{v d}=0.093$. Legend: square $=$ experiment, $\mathrm{x}=$ model prediction.

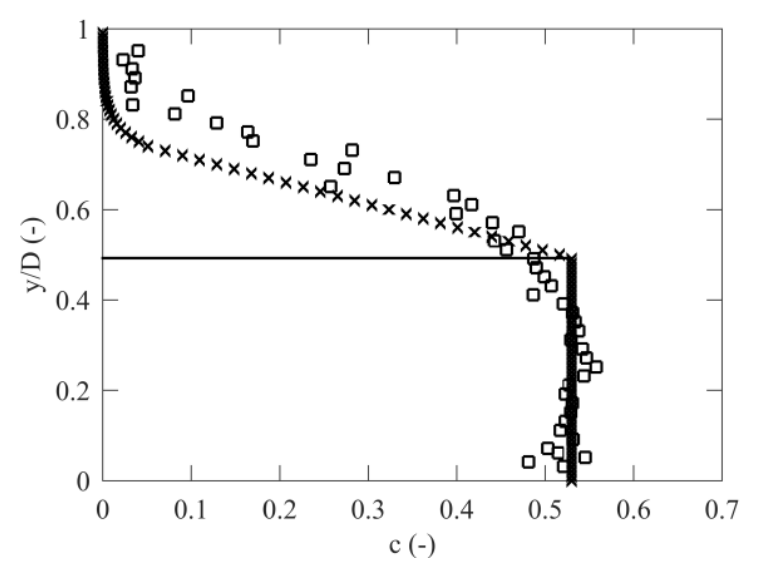

Fig. 4. Distribution of granular concentration at velocity slightly above deposition limit for flow of SP090 slurry $\left(C_{v d, \max }=0.37\right)$ at pipe inclination of $-15 \mathrm{deg}, V_{m}=1.1 \mathrm{~m} \cdot \mathrm{s}^{-1}$, $C_{v d}=0.19$. Legend: square $=$ experiment, $\mathrm{x}=$ model prediction.

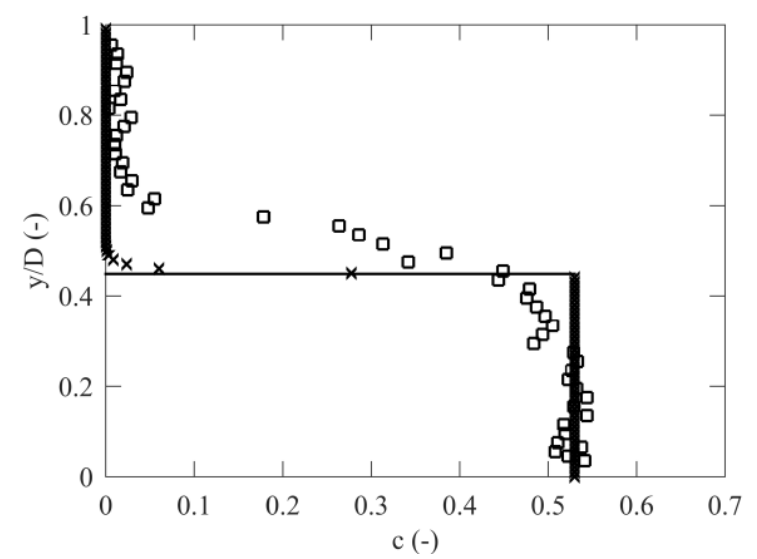

Fig. 5. Distribution of granular concentration at velocity slightly above deposition limit for flow of SP090 slurry $\left(C_{v d, \max }=0.26\right)$ at pipe inclination of $-25 \mathrm{deg}, V_{m}=0.9 \mathrm{~m} . \mathrm{s}^{-1}$, $C_{v d}=0.048$. Legend: square $=$ experiment, $\mathrm{x}=$ model prediction. 


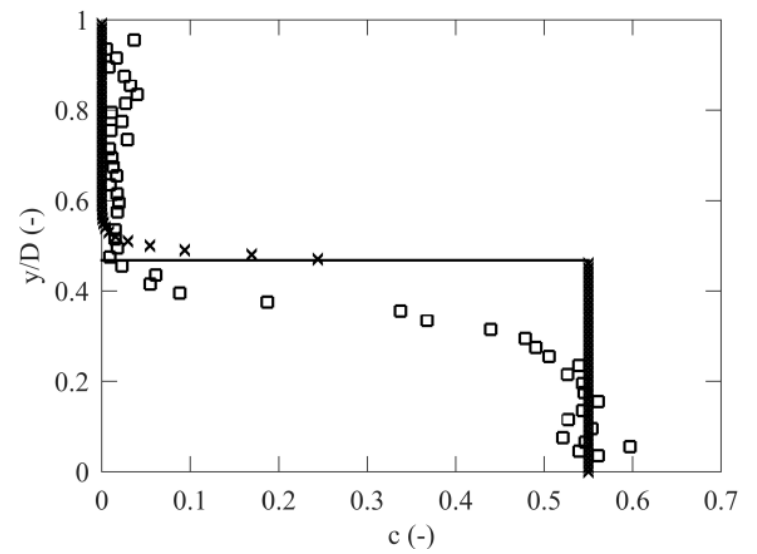

Fig. 6. Distribution of granular concentration at velocity slightly above deposition limit for flow of SP090 slurry $\left(C_{v d, \max }=0.26\right)$ at pipe inclination of $-25 \mathrm{deg}, V_{m}=0.72 \mathrm{~m} \cdot \mathrm{s}^{-1}$, $C_{v d}=0.022$. Legend: square $=$ experiment, $\mathrm{x}=$ model prediction.

The model predicts very well the shapes of granular concentrations for the angle of inclination -15 degrees (Figures 3 and 4), where both the experiment and the model prediction exhibit a developed shear layer above the sliding bed in the pipe. In case of -25 degree, however, the model prediction is less convincing. In fact, the model predicts a virtually fully stratified flow withy negligible thickness of the shear layer. This corresponds with the idea of a fast sliding bed preventing the development of the shear layer. The measured profiles show less sharp interface between the bed and the flow above the bed (Figures 5 and 6). We suspect that this may a consequence of flow instability associated with the caterpillar behaviour observed visually at velocities near the deposition limit during the experiments in the pipe. While the particles move in kind of density waves throughout the loop, the model predicts stable conditions, with almost identical velocities of sliding bed and flowing liquid if the pipe is inclined to -25 degree. Therefore, the shear stress between the two layers is very small and hence the very thin predicted shear layer.

\subsection{Deposition limit velocity}

We use the same layered model with the same combination of model constants and inputs/outputs to predict the deposition limit velocity in negatively slopes pipes and to compare the model predictions with our experimental observations for the SP090-sand slurry flows.

Figure 7 compares the visually observed deposition limit velocities with the ones predicted by the model for negatively inclined flows. The experiments demonstrated a strong effect of the negative slope on the deposition limit velocity, $V_{D}$. Values of $V_{D}$ tended to diminish with the increasing negative slope. It is not surprising if one realizes that $V_{D}$ must tend to zero as the slope approaches the slip-point angle of the bed sliding above which it slides due to gravity even without any support by the flow.

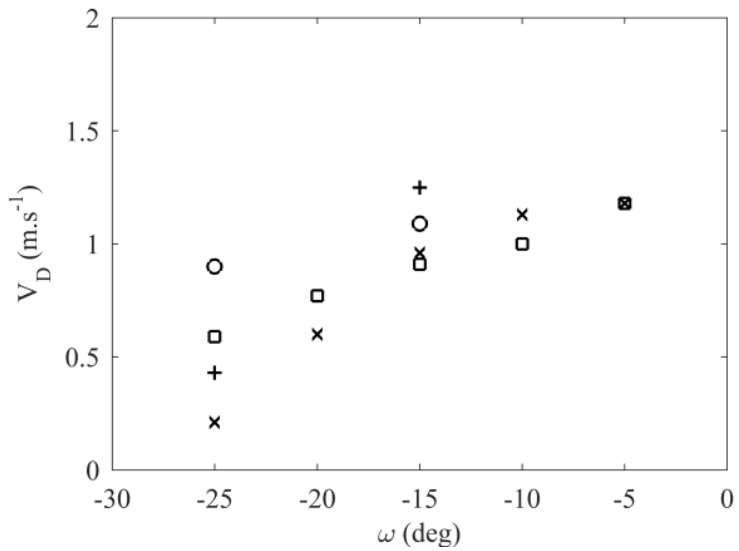

Fig. 7. Deposition limit velocity of SP090 slurry flow in pipe at different inclination angles. Legend: $\mathrm{o}=$ experiment $\left(C_{v d, \max }=0.26\right),+=$ prediction by layered model $\left(C_{v d, \max }=0.26\right), \quad$ square $=$ experiment $\left(C_{v d, \max }=0.37\right)$, $\mathrm{x}=$ prediction by layered model $\left(C_{v d, \max }=0.37\right)$

What may be surprising is the finding that the layered model predicts even bigger effect, i.e. stronger reduction of $V_{D}$, than observed if the inclination angle exceeds say -20 degrees (Figure 7). This may be due to the model inability to the flow instabilities in steep flows into account.

One additional limitation of the layered model to predict $V_{D}$ must be discussed. It is impossible to predict $V_{D}$ by the model if it predicts fully stratified flow for the given conditions. The reason is simple. If all grains are transported in the sliding bed (no transport outside the sliding bed) it is impossible to reach the threshold where the bed stops sliding (i. e. $V_{D}$ ) and in the same time maintain $C_{v d}>0$ as required by the model input. Already at flow velocities slightly above the deposition limit, where the bed slides very slowly, the model generates an unrealistically thick bed to satisfy a required value of $C_{v d}$

\section{Conclusions}

Our investigation of negatively sloped flows of settling slurry (the $0.87-\mathrm{mm}$ narrow-graded sand in water) near and at the deposition limit velocity in a $100-\mathrm{mm}$ pipe showed that

- the solids distribution in the tested partially stratified slurry flow is very sensitive to the pipe inclination; the maximum stratification (virtually fully stratified flow) is reached at inclination angles around 30 degree;

- our layered model for inclined settling slurries captures the variation in the flow stratification at velocities slightly above the deposition limit velocity very well, particularly if the slope is milder than -20 degrees;

- the deposition limit velocity distribution in the tested partially stratified slurry flow is very sensitive to the pipe inclination; it diminishes strongly with the steeper negative slope of the pipe and tends to zero for inclination angles exceeding say -30 degrees; 
- the layered model captures the variation in the deposition limit velocity with flow inclination well, particularly if the slope is milder than - 20 degrees; the model is not able to predict the deposition limit if the flow is fully stratified and the input delivered concentration does not tend to zero.

The research has been supported by the Czech Science Foundation through the grant project No. 17-14271S.

\section{References}

1. V. Matoušek, M. Kesely, P. Vlasák, EPJ Web Conf., Volume 180 (2018)

2. V. Matoušek, J. Krupička, P. Vlasák, Powder Technology, s. 317-326 ISSN 0032-5910 (2018),

3. V. Matoušek, J. Krupička, V. Pěník, Particul. Sci. Technol. 32(2), 186-196 (2014)

4. J. Krupička, V. Matoušek, Journal of Hydrology and Hydromechanics, 62(2), 126-132 (2014) 